\title{
Probabilistic evaluation of interconnectable capacity for wind power generation
}

\section{AUTHOR(S):}

Kashima, Kenji; Kato, Masakazu; Imura, Jun-ichi; Aihara, Kazuyuki

\section{CITATION:}

Kashima, Kenji ... [et al]. Probabilistic evaluation of interconnectable capacity for wind power generation. The European Physical Journal Special Topics 2014, 223(12): 24932501

ISSUE DATE:

2014-09-26

URL:

http://hdl.handle.net/2433/200198

\section{RIGHT:}

The final publication is available at Springer via http://dx.doi.org/10.1140/epjst/e201402276-9.; この論文は出版社版でありません。引用の際には出版社版をご確認ご利用くだ さい。; This is not the published version. Please cite only the published version. 
EPJ manuscript No.

(will be inserted by the editor)

\title{
Probabilistic evaluation of interconnectable capacity for wind power generation
}

\section{Stochastic linearization approach}

\author{
Kenji Kashima $^{1, a}$, Masakazu Kato ${ }^{2, b}$, Jun-ichi Imura ${ }^{3, c}$, and Kazuyuki Aihara ${ }^{4, d}$ \\ 1 Kyoto University, 36-1, Yoshida Honmachi, Sakyo-ku, Kyoto, Japan 606-8501 \\ 2 Tokyo Denki University, 5, Senju-Asahi-cho, Adachi-ku, Tokyo, Japan 120-8551 \\ 3 Tokyo Institute of Technology, 2-12-1, O-okayama, Meguro, Tokyo, Japan 145-0062 \\ 4 The University of Tokyo, 4-6-1, Komaba, Meguro, , Tokyo, Japan 153-8505
}

\begin{abstract}
We propose a novel framework for probabilistic evaluation of interconnectable capacity for wind power generation. This is based on mathematical modeling of load frequency control systems, which captures their nonlinear (saturation and rate limiting) dynamical characteristics, and stochastic uncertainty of wind power output deviation. The method called stochastic linearization is used to analyze resulting power quality. The effectiveness of the proposed method is examined by numerical simulation.
\end{abstract}

\section{Introduction}

Recently, renewable energies are introduced all over the world as one of the promising measures against global warming. Such renewable energies include solar power, wind power and other natural energy resources. Solar power and wind power, however, are sensitively dependent on weather conditions, which may bring about power quality deterioration upon large penetration to the power system. Many European countries like Germany and Spain are actively promoting to introduce wind power and solar power generation [1]. Also in Japan [2], the government is promoting to introduce wind power and solar power (photovoltaic). However, Japanese power companies, except for major ones, have the maximum generation capacities of wind power to be interconnected as shown in the Table 1, which are revised based on actual wind power data. This limitation comes from Japanese power network characteristics. The Japanese power network has two power frequencies, $50[\mathrm{~Hz}]$ in the eastern part and $60[\mathrm{~Hz}]$ in the western part. Both networks are interconnected via frequency conversion stations of total capacity of 1200 [MW]. Due to the landform of Japan, each power network is generally interconnected by only one transmission route with its neighboring network [3] although each power network has loop configurations. This also comes from the Japanese power system policy that each power network keeps

\footnotetext{
a e-mail: kashima@amp.i.kyoto-u.ac.jp

b e-mail: kato@eee.dendai.ac.jp

c e-mail: imura@mei.titech.ac.jp

d e-mail: aihara@sat.t.u-tokyo.ac.jp
} 
Table 1. Maximum wind power generation capacity of Japanese power companies. Tokyo, Chubu, and Kansai have not decided the maximal capacity.

\begin{tabular}{ll}
\hline Electric Power Company & Capacity [MW] \\
\hline Hokkaido E.P.C. & 360 \\
Tohoku E.P.C. & 1180 \\
Tokyo E.P.C. & - \\
Hokuriku E.P.C. & 150 \\
Chubu E.P.C. & - \\
Kansai E.P.C. & - \\
Chugoku E.P.C. & 620 \\
Shikoku E.P.C. & 250 \\
Kyushu E.P.C. & 1000 \\
Okinawa E.P.C. & 25 \\
\hline
\end{tabular}

load/generation balance within the individual network and that interconnection lines are used for stable and economic power exchange and emergency operation. Thus, load/generation unbalance with intermittent power supply like wind power brings about large power flow fluctuation on the interconnection lines. This disturbs stable and economic power system operation. This is the reason for each power system to have the maximum capacity of wind power generation to be interconnected.

Currently, an algebraic method, approved by the Japanese Governmental committee in 2005, is used to decide the maximum capacity of wind power generation [2]. However, this method does not consider the dynamic property of a power system like generating plant characteristics. In addition, feed-in tariff (FIT) introduced in July, 2012 accepts disconnection of renewable generation from the network to attenuate fluctuation. Therefore, it is important to estimate the probability of this disconnection, rather than to know whether or not it can happen. This motivates us to shift from the worst case analysis to a probabilistic evaluation of power quality deterioration caused by introducing wind energy.

In view of these circumstances, in this paper we propose an advanced method to decide the maximum generation capacity of wind power generation that incorporates power system dynamics and a probabilistic approach. To this end, stochastic linearization [5], which is a powerful tool to analyse stochastic systems with weak nonlinearity as described in Section 3, is used to analyze wind power control systems; see also prelminary results by the authors $[8,9]$. From different motivations, this technique was also used by Guo et al. [7] to analyze wind turbine control systems taking the saturation effect ignored in [6] into account.

This paper is organized as follows: In Section 2, we provide our mathematical model for power networks interconnected to wind power generation. In Section 3, theoretical background for linearization as well as its algorithm are given. In Section 4, we illustrate a numerical example. Finally, Section 5 concludes this paper.

\section{Mathematical model of Load Frequency Control Systems}

\subsection{Load Frequency Control}

Due to the Japanese power system policy mentioned in Section 1, the maximum capacity of wind power generation for a power network is decided assuming no compensation by other interconnected power networks, although each power network is interconnected. In addition, a simple model with an equivalent single generator (a 


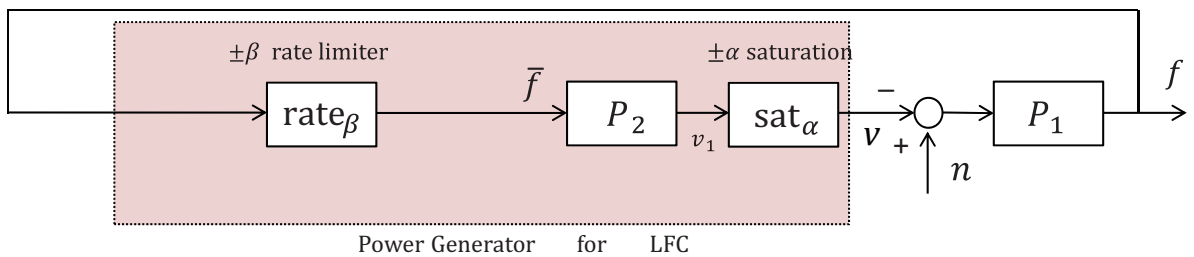

Fig. 1. Block diagram of a load frequency control model.

lumped generator) is widely used for the evaluation of frequency deviation caused by load/generation unbalance. Figure 1 depicts the mathematical model of a single power network with a load frequency control at the central load dispatching office. A load frequency control applied here is FFC (Flat Frequency Control) considering the aforementioned Japanese power system policy. Generation output adjustment proportional to frequency deviation is controlled at the power plant in this model. First, the input $n$ represents fluctuation of a wind power generation output, whose characteristics are specified in the next subsection. Next, the frequency deviation $f$, which is the output of the system $P_{1}$, caused by the total power deviation $n-v$ is modeled by

$$
T_{1} \dot{f}(t)=f(t)+(n(t)-v(t))
$$

where $T_{1}$ specifies the low pass property due to its physical inertia, e.g., the load characteristics and system inertia of the power system. In this paper, the standard deviation of $f$ is regarded as a measure for the resulting power quality. This signal is fed back to the load frequency control system (LFC) to compensate the fluctuation. The compensator consists of thermal power plants whose power generation output is adjustable in order to cancel the renewables output fluctuation. Actually, the dynamical property of a power generator for LFC is characterized as

$$
T_{2} \dot{v}_{1}(t)=v_{1}(t)+\bar{f}(t), \bar{f}(t)=\operatorname{rate}_{\beta}(f(t)), v(t)=\operatorname{sat}_{\alpha}\left(v_{1}(t)\right) .
$$

It should be emphasized that there are two physically strict constraints for the ability of these power plants. One is that there are upper and lower bounds for the generation output. We model this limited capacity by (static) saturation to $[-\alpha, \alpha]$ as follows:

$$
\operatorname{sat}_{\alpha}(x):= \begin{cases}-\alpha, & \text { for } x<-\alpha \\ x, & \text { for }|x| \leq \alpha \\ \alpha, & \text { for } x>\alpha\end{cases}
$$

The other is that we cannot change thermal power plants output arbitrarily quickly. To incorporate this limited responsiveness, we employ the rate limiter to $[-\beta, \beta]$, which restricts the slope of the output signal to this range. Therefore, $-v$ represents the power adjustment in LFC output to attenuate the frequency deviation.

\subsection{Wind power deviation}

We proceed to modeling of the wind power output deviation $n$. In this study, we assume that the wind power is not a single local one, but a large scale, widely-spread one. In such a situation, though there still is fluctuation, we can expect smoothing effect by aggregation, which has been observed from real data in Japan [2]. Thus, it 


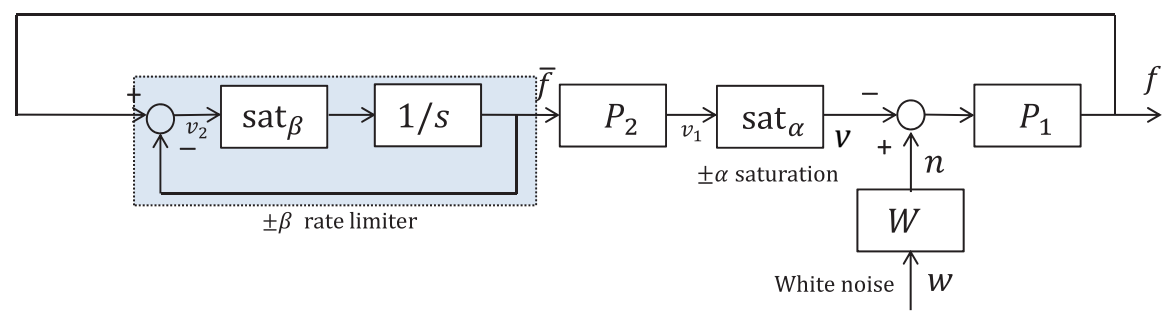

Fig. 2. Block diagram of a load frequency control model with an approximate rate limiter.

is reasonable to assume the high frequency component of fluctuation $n$ decay quickly. Such frequency-domain decay characteristics can be modeled by

$$
\dot{n}(t)=B n(t)+A w(t),
$$

where $w(t)$ is the white noise with a uniform power spectrum; see Fig. 2. The parameters $A$ and $B$ specify the scale and the frequency distribution, respectively.

As for the rate limiter, it is standard to approximate it as the feedback loop consisting of an integrator and saturation as in Fig. 2:

$$
\dot{\bar{f}}(t)=\operatorname{sat}_{\beta}\left(v_{2}(t)\right), v_{2}(t)=f(t)-\bar{f}(t) .
$$

In summary, the dynamics is represented by linear systems and two saturation blocks. Our goal is to evaluate the standard deviation of output $f$, when the input is given by white noise. For this purpose, we employ stochastic linearization summarized in the next section.

For later use, we define transfer functions of three linear dynamics as follows:

$$
W(s):=\frac{A}{s+B}, P_{i}(s):=\frac{1}{T_{i} s+1}(i=1,2) .
$$

These rational functions describe frequency domain input-output characteristics, e.g.,

$$
[\mathcal{F} n](\omega)=W(\mathrm{i} \omega) \cdot[\mathcal{F} w](\omega)
$$

where $\mathcal{F}$ denotes the Fourier transform.

\section{Probabilistic evaluation method}

\subsection{Stochastic linearization}

It is known [4] that when we inject white noise to a stable linear system, the probability distribution of any output signals converges to a normal distribution. However, this is not true for nonlinear systems. The main idea of stochastic linearizaiton [5] is to approximate the saturations by an appropriate (static linear) gain under the assumption that each non-Gaussian stationary distribution is sufficiently close to the normal one. Suppose that random variable $v$ has normal distribution $\mathcal{N}\left(0, \sigma_{v}^{2}\right)$, that is, the probability density function is given by

$$
\begin{aligned}
& \operatorname{Prob}[v \in A]=\int_{A} \phi_{\sigma_{v}}(z) d z, \\
& \phi_{\sigma}(z):=\frac{1}{\sqrt{2 \pi} \sigma} \exp \left(-\frac{z^{2}}{2 \sigma^{2}}\right),
\end{aligned}
$$




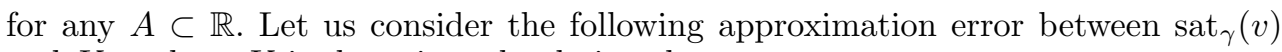
and $K v$, where $K$ is the gain to be designed:

$$
\mathrm{E}\left[\left(\operatorname{sat}_{\gamma}(v)-K v\right)^{2}\right] .
$$

It is known [5] that the gain $K$ minimizing this error is given by $K\left(\sigma_{v}, \gamma\right)$, where

$$
K(\sigma, \gamma):=\int_{-\gamma}^{\gamma} \phi_{\sigma}(z) d z .
$$

Note that $K(\sigma, \gamma) \in[0,1]$ for any $\sigma, \gamma>0$.

An important feature of this approximation is that the obtained gain depends on both the saturation level $\gamma$ and also the standard deviation $\sigma_{v}$ of the input random variable.

\subsection{Algorithm}

When we apply this approximation to sat ${ }_{\alpha}$ and $\operatorname{sat}_{\beta}$ in Fig. 2, we need to know the stationary probability distributions of $v_{1}$ and $v_{2}$, respectively. In control theory ([4]), it is known when we add white noise to a stable linear system with transfer function $G(s)$, the output probability distribution converges to $\mathcal{N}\left(0,\|G\|_{2}^{2}\right)$ where $\|\cdot\|_{2}$ denotes the $H^{2}$-norm defined by

$$
\|G(s)\|_{2}:=\sqrt{\int_{-\infty}^{\infty}|G(\mathrm{i} \omega)|^{2} d \omega .}
$$

This fact implies that if sat ${ }_{\alpha}$ and sat ${ }_{\beta}$ can be replaced by simple gains $K_{\alpha}$ and $K_{\beta}$, then the stationary probability distributions of $v_{1}$ and $v_{2}$ are $\mathcal{N}\left(0,\left\|G_{w v_{1}}\right\|_{2}^{2}\right)$ and $\mathcal{N}\left(0,\left\|G_{w v_{2}}\right\|_{2}^{2}\right)$, where $G_{w v_{i}}$ represents the transfer function from $w$ to $v_{i}$

$$
\begin{aligned}
G_{w v_{1}} & :=W \frac{K_{\beta} P_{1} P_{2}}{s+K_{\beta}+P_{1} P_{2} K_{\alpha} K_{\beta}}, \\
G_{w v_{2}} & :=W \frac{s P_{1}}{s+K_{\beta}+P_{1} P_{2} K_{\alpha} K_{\beta}} .
\end{aligned}
$$

These functions can be obtained through trivial algebraic calculation based on relationships like (7).

Recall that we have not yet discussed how to decide $K_{\alpha}$ and $K_{\beta}$. Following the method in Section 3.1, we can expect a good approximation accuracy when

$$
\begin{aligned}
& K\left(\sigma_{v_{1}}, \alpha\right)=K_{\alpha}, \\
& K\left(\sigma_{v_{2}}, \beta\right)=K_{\beta}
\end{aligned}
$$

hold, where $\sigma_{v_{i}}$ denotes the stationary standard deviation of $v_{i}$. Combining these relations, we solve the following equations:

$$
\begin{aligned}
& K\left(\left\|G_{w v_{1}}\right\|_{2}, \alpha\right)=K_{\alpha}, \\
& K\left(\left\|G_{w v_{2}}\right\|_{2}, \beta\right)=K_{\beta},
\end{aligned}
$$

with respect to $K_{\alpha}$ and $K_{\beta}$, then we adopt them as a linearizing gain. 
After the saturations are suitably linearized, i.e., $K_{\alpha}$ and $K_{\beta}$ are determined, the stationary probability distribution of $f$ is approximated by $\mathcal{N}\left(0,\left\|G_{f}\right\|_{2}^{2}\right)$ where $G_{f}$ is the transfer function from $w$ to $f$

$$
G_{f}:=W \frac{k\left(s+K_{\beta}\right) P_{1}}{s+K_{\beta}+P_{1} P_{2} K_{\alpha} K_{\beta}} .
$$

Thus, $\left\|G_{f}\right\|_{2}$ will be a good measure for the resulting power quality.

We give some comments on the computational complexity for solving these equations. First, the optimal gain in (11) and the $H^{2}$-norm in (12) can easily be obtained, since their calculation reduces to the evaluation of the error function and solving a linear equation of the same size as the system degree, respectively. Furthermore, $K_{\alpha}$ and $K_{\beta}$ are contained in [0,1], which is trivial since these gains are obtained by approximately linearizing the saturation function whose slope is normalized as shown in (3). Therefore, the solution to (17) and (18) can be easily found numerically.

Note also that since the dynamics are nonlinear, the relation between $n$ and $f$ is not linear either. That is, when we multiply $W(s)$ by constant $C$, the estimated stationary standard deviation of $f$ is not necessarily multiplied by $C$ because the solution to (17) and (18) change; see also examples in the next section. To put it differently, thermal plants equipped with the same capacity $(\alpha$ and $\beta$ ) have various actual performance $\left(K_{\alpha}\right.$ and $\left.K_{\beta}\right)$ depending on the wind power generation output scale. This enables us to perform cost-benefit analysis for the capacity $\alpha$ and $\beta$ of thermal plants.

We make a brief comment on the restriction of the proposed method. In the framework above, we applied normal distribution approximation upon wind power deviation modeling and stochastic linearization for the sake of simplicity, and then estimated the 2nd-order moments only. However, more detailed statistical characteristics are necessary to capture the real behavior of several important phenomena such as turbulence [11]. Thus, we need to be careful when we apply the proposed method to real situations.

\section{Numerical example}

In this section, we evaluate the effectiveness of the proposed method through numerical simulation.

First, considering the spectrum analysis result of the real data of the wind power generation output $([8])$, we take

$$
B=0.001
$$

Concerning the other parameter, we rely on the experimental fact $([10])$ that the deviation in 20-min windows is within $20 \%$ of the interconnected wind power amount with a probability higher than $99.7 \%$. Based on this fact and a prior Monte Carlo simulation, we fix

$$
A=0.0021 \times L
$$

for the case where we interconnect $L[\mathrm{MW}]$ of wind power. Figure 3 shows sample paths for $n(t)$ with $L=650[\mathrm{MW}$. We can see that the difference between highest and lowest values in any 20-min windows is smaller than $650 \times 0.20=130[\mathrm{MW}]$ in each path. The linear time invariant systems in Fig. 1 are given by

$$
T_{1}=3, T_{2}=1 / 0.15 \text {. }
$$


Table 2. Estimated standard deviation of frequency fluctuation and approximated gains versus interconnected wind power generation.

\begin{tabular}{cccc}
\hline $\begin{array}{c}\text { Interconnected wind power } \\
L[\mathrm{MW}]\end{array}$ & $\begin{array}{c}\text { Estimated SD } \\
f / k[\mathrm{~Hz}]\end{array}$ & $K_{\alpha}$ & $K_{\beta}$ \\
\hline 500 & 0.0486 & 0.9640 & 0.0583 \\
550 & 0.0542 & 0.9406 & 0.0481 \\
600 & 0.0602 & 0.9117 & 0.0403 \\
650 & 0.0665 & 0.8788 & 0.0341 \\
700 & 0.0732 & 0.8418 & 0.0292 \\
750 & 0.0802 & 0.8037 & 0.0252 \\
800 & 0.0876 & 0.7650 & 0.0219 \\
\hline
\end{tabular}

For the LFC capacity, we assume the system capacity of 2500 [MW] (system constant $k=250[\mathrm{MW} / \mathrm{Hz}])$. Taking the maximum width of the output adjustment range as $2 \%$ of $2500[\mathrm{MW}]$, we choose

$$
\alpha=25[\mathrm{MW}]
$$

Concerning the rate limiter, we suppose that the capacity of the thermal power plant is $500[\mathrm{MW}$, and that the maximal acceptable change rate is $2 \% / \mathrm{min}$, that is, 10 $[\mathrm{MW} / \mathrm{min}]$ that leads to

$$
\beta=0.166[\mathrm{MW} / \mathrm{sec}] .
$$

Finally, the frequency deviation is

$$
f / k[\mathrm{~Hz}] \text {. }
$$

Table 2 shows the estimated standard deviation of $f / k$ for various $L$. The results obtained by Monte Carlo simulation (time discretization $d t=0.5, t=0 \sim 2 \times 10^{6}$ [sec]) were $0.0598,0.0662$, and 0.0773 for $L=600,650$, and 700 , which show the high accuracy of the estimation obtained by the proposed method. In the Japanese electricity network, it is a usual requirement that the frequency deviation should be within $\pm 0.3[\mathrm{~Hz}]$. We assume that the load fluctuation is supposed to bring about deviation within $\pm 0.1[\mathrm{~Hz}]$. This means that we can accept frequency deviation within $\pm 0.2[\mathrm{~Hz}]$ caused by wind power uncertainty. Then, by the $3 \sigma$-rule, we can estimate that

$$
L=650[\mathrm{MW}]
$$

is the maximal interconnectable wind power. Note that $K_{\alpha}$ and $K_{\beta}$ are monotonically decreasing functions of $L$. This means that these saturation components act as lower gains when interconnected to a larger amount of wind power generation.

We are interested in when and how load frequency control capacity $\alpha$ and $\beta$ contribute to the power quality. Thus, Fig. 4 shows the result for various $\alpha$ and $\beta$ for $L=700$. In this figure, 'No control' means the performance without the load frequency control, that is, $\alpha=\beta=0$. On the other hand, 'No limitation' means the performance with unbounded thermal plant capacity, that is, $\alpha \rightarrow+\infty$, and $\beta \rightarrow+\infty$. These values are given by $\left\|G_{f}\right\|_{2} / k$ with $K_{\alpha}=K_{\beta}=0$ and $K_{\alpha}=K_{\beta}=1$, between which the performance for all $\alpha$ and $\beta$ lies. From this result, we can estimate that the total amount $\alpha$ of the thermal plant is more significant than the responsiveness $\beta$ in this range of parameters. 


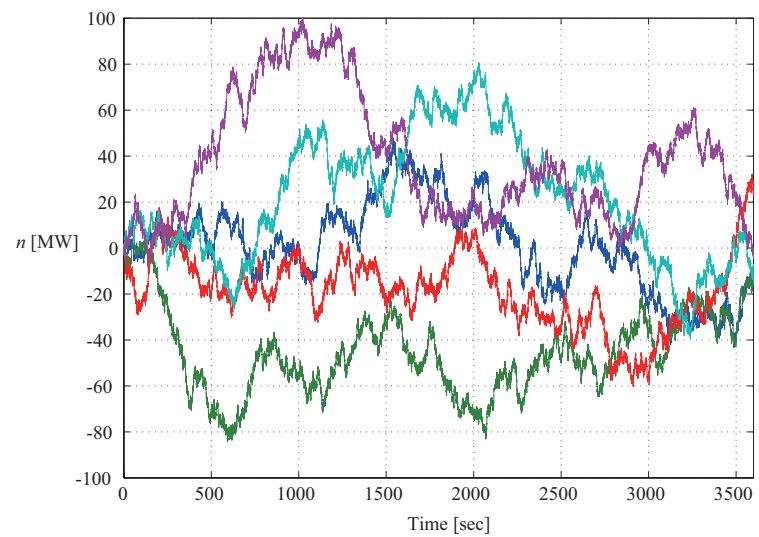

Fig. 3. Sample paths for noise $n(t)$ with $L=650$.

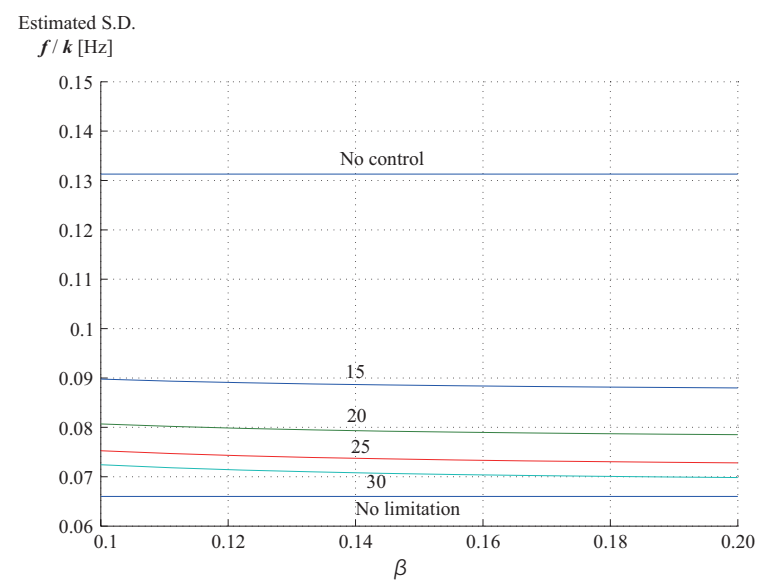

Fig. 4. Estimated standard deviation of frequency fluctuation for $L=700, \alpha=$ $15,20,25,30$ and $\beta \in[0.1,0.2]$.

\section{Conclusion}

In this paper, we proposed a framework for a probabilistic evaluation of interconnectable capacity for wind power generation. We emphasize the following advantages of the proposed method over existing ones:

- the proposed method takes the dynamical property of wind power deviation and load frequency control systems into explicit account,

- the estimated power quality (the stationary standard deviation of $f$ ) depends on $\alpha$ and $\beta$, whose contribution is ignored when we apply standard linearization (the Jacobian matrix at the origin), and

- the evaluation is not a worst case analysis but a probabilistic one.

The ongoing further investigation contains

- we additionally take demand deviation into account. This deviation should not be characterized in the frequency domain as in Section 2.

- we also need more detailed discussion for the parameter determination in the wind deviation model to suitably describe individual situations. It should be emphasized 
that the proposed scheme is applicable to the case where $W, P_{1}$, and $P_{2}$ have higher order dynamics.

\section{Acknowledgments}

This research is supported by the Aihara Innovative Mathematical Modelling Project, the Japan Society for the Promotion of Science (JSPS) through the Funding Program for World-Leading Innovative R\&D on Science and Technology (FIRST Program)," initiated by the Council for Science and Technology Policy (CSTP).

\section{References}

1. "Wind in power 2011 European statistics," European Wind Energy Association (2012)

2. http://www.escj.or.jp/energy/wg/pdf/report_windpower_wg.pdf (in Japanese)

3. Electric Power System Council of Japan: http://www.escj.or.jp/english/status/images/01.pdf

4. K. Zhou, J. Doyle, and K. Glover Robust and Optimal Control (Prentice Hall, 1995)

5. S. Ching, Y. Eun, C. Gokcek, P. Kabamba, and S. Meerkov Quasilinear Control: Performance Analysis and Design of Feedback Systems with Nonlinear Sensors and Actuators (Cambridge University Press, 2011)

6. Y. Guo, W. Wang, C. Y. Tang, J. N. Jiang, and R. G. Ramakumar, Proc. American Control Conference (2013), pp. 2896-2903.

7. Y. Guo, P. T. Kabamba, S. M. Meerkov, H. R. Ossareh, and C. Y. Tang, Proc. IEEE Conference on Decisions and Control (2013), pp. 1307-1312.

8. K. Kashima, M. Kato, J.-I. Imura, and K. Aihara, Proc. Annual Conf. Power and Energy Society, IEE Japan (2013), pp. 155-156 (in Japanese)

9. M. Kato, K. Kashima, J.-I. Imura, and K. Aihara, Workshop on Resilient Power Grids and Extreme Events, Potzdam (2013)

10. Final report of the Working Group on "Assessment of maximum wind power generation capacity to be interconnected" Electric Power System Council of Japan (2012)

11. P. Milan, M. Wächter, and J. Peinke, Physical Review Letters (2013), vol. 110, issue 13, 138701. 